\title{
A MicroRNA-124 Polymorphism is Associated with Fracture Healing via Modulating BMP6 Expression
}

\author{
Lin Zou $^{\mathrm{a}}$ Guichun Zhang ${ }^{\mathrm{a}}$ Lifeng Liu ${ }^{\mathrm{a}}$ Chen Chen ${ }^{\mathrm{a}}$ Xuecheng Cao ${ }^{\mathrm{a}}$ Jinfang Cai ${ }^{\mathrm{a}}$ \\ aDepartment of Traumatic Orthopedic Surgery, the General Hospital of Jinan Military Command, Jinan, \\ China
}

\section{Key Words}

MicroRNA-124 • rs531564 • Polymorphism • Fracture healing • BMP6 • Apoptosis

\begin{abstract}
Background: miR-124-3p has been reported to be involved in the pathogenesis of many diseases by modulating a variety of signaling pathways. In this study, we aimed to understand the impact of miR-124-3p expression level on the fracture healing in the patients of metaphyseal fracture of distal tibia, who received minimal invasive percutaneous plate osteosynthesis. Methods: We firstly collected 195 patients of metaphyseal fracture of distal tibia, and the genotype of rs531564 was determined: GG $(n=124)$ and $G C+C C(n=71)$. We collected information of the participants including age, gender, total in-hospital time, smoking and alcohol consumption. Subsequently, we searched the miRNA database online to identify the possible binding sequence of miR-124-3p located within the $3^{\prime}-$ UTR of the target gene. We did correlation analysis and luciferase to understand the regulatory relationship between miR124-3p and BMP6. Meanwhile, we also conducted real time PCR and western blotting analysis to study the mRNA and protein expression level of BMP6 in different genotype groups. We then treated the cells with scramble control, miR-124-3p mimics, BMP6 siRNA and miR-124$3 p$ inhibitors to investigate the influence of miR-124-3p on the expression of BMP6, viability and apoptosis of cells. Results: Total in-hospital time was significantly longer in GC+CC group than GG group. MiR-124-3p was up-regulated in GG group than GC and CC groups. BMP6 was virtual target of miR-124-3p. There existed negative regulatory relationship betweenmiR124-3p and BMP6. The mRNA and protein expression level of BMP6 decreased in GG group. MiR-124-3p decreased the expression of BMP6. MiR-124-3p negatively interfered with the viability of cells and BMP6 positively interfered with the viability of cells. MiR-124-3p reduced apoptosis and BMP6 promoted apoptosis. Conclusion: These data proved the expression of miR-124-3p was associated with the healing of metaphyseal fracture of distal tibia, and could be recognized as a biomarker to predict the healing of metaphyseal fracture of distal tibia.
\end{abstract}




\section{Cellular Physiology Cell Physiol Biochem 2017;41:2161-2170 \begin{tabular}{l|l|l} 
and BiOChemistry & Dublished ontine: April 21, 2017 & $\begin{array}{l}\text { C } 2017 \text { The Author(s). Published by S. Karger AG, Basel } \\
\text { www.karger.com/cpb }\end{array}$
\end{tabular} \\ Zou et al.: Rs531564 Polymorphism is Associated with Fracture Healing}

\section{Introduction}

Fractures are among the most common traumatic injuries in humans and osteoporosis related fractures are the fastest growing health care problem of aging [1]. The vast majority of fractures sustained worldwide are either untreated (e.g. patients in many third-world countries) or are treated in a manner that involves a periosteal response and a process of endochondral bone formation [2]. Despite the intrinsic ability of the body to heal fractures, patient risk factors can significantly impair skeletal repair [3]. The rate of delayed fracture healing or non-union is highest amongst subpopulations with specific risk factors such as smoking, advanced age, steroid use, use of certain pharmaceuticals (i.e. anti-cancer drugs) and metabolic diseases such as diabetes mellitus (DM) [3]. An increased mechanistic understanding for impaired osseous healing associated with specific high-risk populations will provide fundamental information necessary to design a regenerative approach for fracture patients with specific risk factors for non-union.

A variety of genetic and environmental factors have been reported to play an important role in the control of the apoptosis of osteoblasts, which is believed to be involved in the determination of fracture healing $[4,5]$. Upregulation of BclXL in osteoblasts surpressed the apoptosis of the host cells, and promoted the regeneration or the strength of the bone [4]. Some other apoptosis repressors were also found to participate in the control of osteogenic differenetiation of osteoblast cells, and zoledronate administration could enhance the survival of osteoblasts and positively contributed to the regeneration of the bone tissue [5]. On the other hand, induction of the apoptosis of osteoblasts could consistently compromised the osteogenesis, and IL-1A treatment could promote the apoptosis of osteoblasts through JNK and p38 MAPK signaling pathway [6]. Meanwhile, the apoptosis of other cell types in bone is also considered to be part of osteogenesis [7]. Apoptosis of chondrocytes is associated with secretion of osteogenic growth factors such as transforming growth factor $\beta$ (TGF- $\beta$ ) and angiogenic factors such as fibroblast growth factor 2 (FGF-2) and may be important in regulating the early steps in bone formation as cartilage matrix is resorbed $[7,8]$.

MicroRNAs are non-protein-coding RNAs which are involved in gene regulation at the post-transcriptional level [9]. First discovered as critical regulators of developmental timing in Caenorhabditis elegans, micro RNAs have since been found to function in almost all cellular processes in animals and plants [10-12]. MicroRNAs are approximately 22 nucleotides in length, generated from large primary transcripts that form imperfect stem loop structures. MicroRNAs regulate gene expression by binding to target mRNAs and promoting transcript degradation or inhibiting translation [13].

A variant (rs531564) located in the miR-124 has been found to interfere with the mature processing of the miRNA, resulting in a reduced expression of mature miRNA. On the other hand, BMP6 is believed to be functionally involved in the control of fracture healing by regulating the apoptosis of osteoblasts, and we also identified BMP6 as a virtual target of miR-124 using in-silicon analysis. In this study, we confirmed the regulatory relationship between miR-124 and BMP6 in OS-732 cells, and studied the association between miR-124 rs531564 polymorphism and fracture healing [14].

\section{Materials and Methods}

Sample collection

A total of 195 patients with metaphyseal fracture of distal tibia who have received surgical intervention in the Department of Traumatic Orthopedic Surgery, the General Hospital of Jinan Military Command (Jinan, China) were enrolled in this study during Sept 2013 and Nov 2014, and all signed written consent prior to donation of $5 \mathrm{ml}$ peripheral blood. Among them, bone tissue samples were collected from 62 patients, and the samples were stored in $-80^{\circ} \mathrm{C}$ for future use. The study protocols were approved by the Ethics Committee of the General Hospital of Jinan Military Command. 


\section{Cellular Physiology Cell Physiol Biochem 2017;41:2161-2170 \begin{tabular}{l|l} 
and Biochemistry Published online: April 21, 2017 & $\begin{array}{l}\text { (c) } 2017 \text { The Author(s). Published by S. Karger AG, Basel } \\
\text { www.karger.com/cpb }\end{array}$
\end{tabular}

\section{Genotyping by taqman genotyping kit}

Qiagen Genomic DNA extraction kits (Valencia, CA, USA) were used to extract DNA from leucocytes according to protocol. Taq-Man genotyping technology (Applied Biosystems, Foster city, CA) was used to perform the SNP genotyping (rs531564) based on the instruction from the supplier. Taqman Genotyper software (Applied Biosystems, Foster city, CA) was used to analyze the genotype calling. All experiments were performed at least three times.

RNA isolation and real-time PCR

TRIzol Reagent (Invitrogen, Carlsbad, CA, USA) was used to isolate the whole RNA from OS-732 cells according to the manufacturer's description. Genomic DNA contamination was excluded using the RNasefree DNase (Promega, Madison, WI, USA), and NANODROP 2000 spectrophotometer (Thermo Scientific, Waltham, MA, USA) was used to quantify the concentration of the RNA as described before. Agarose gel electrophoresis was used to examine the integrity of the RNA with values of A260/A280 from 1.8 to 2.0 were accepted. The DNase-treated total RNAs was subjected to reverse transcribed with oligo primer (Takara, Japan) containing the M-MLV Reverse Transcriptase (Promega, Madison, WI, USA) in accordance with manufacturer's recommendation. CFX96TouchTM Real-Time PCR Detection System (Bio-Rad, USA) with FastStart Essential DNA GreenMaster (Roche, Indianapolis, IN, USA) was used to detect the levels of BMP6 mRNA and miR-124-3p using the primer sets: BMP6, 5'-_CCGTGTAGTATGGGCCTCAGA-3' and 5'-TCACAACCCACAGATTGCTAGTG-3'. miR-124-3p, 5'- CTAGCCTGCAGGAGCTGCGGTAGGAAGGATGC-3' and 5'- ATCCGGCCGGCCCAGGCTATTTTCTGCCGCCG-3'. The cycle parameters were set up at $95^{\circ} \mathrm{C}$ for $30 \mathrm{~s}, 40$ cycles of $95^{\circ} \mathrm{C}$ for $5 \mathrm{~s}$ and $60^{\circ} \mathrm{C}$ for $30 \mathrm{~s}$. The house keeping gene (GAPDH for mRNA and U6 for miRNA) was carried out in parallel with all samples to normalize the cDNA loading. The relative mRNA expression for the BMP6 mRNA and miR-124 was determined using the $2^{-\Delta \Delta C T}$ method. All reactions were carried out three times.

\section{Cell culture and transfection}

DMEM medium (Invitrogen, Carlsbad, CA, USA) with $100 \mu \mathrm{g} / \mathrm{mL}$ streptomycin, $100 \mathrm{U} / \mathrm{mL}$ penicillin and $10 \%$ FBS (fetal bovine serum) (Gibco Invitrogen, Grand Island, NY) was used to incubate the OS-732 cells in an atmosphere of $5 \% \mathrm{CO}_{2}$ in air at $37^{\circ} \mathrm{C}$. When the cells were grown to $70-80 \%$ confluence, lipofectamine ${ }^{\mathrm{TM}}$ 2000 transfection reagent (Invitrogen, Carlsbad, CA, USA) was used to perform the transfection following the protocol by the supplier, and we used the fresh medium (DMEM/F12) supplemented 10\% FBS instead of the medium 4 hours after transfection. All experiments were performed in triplicate.

\section{Cell proliferation assay}

The MTT [3-(4, 5-dimethylthiazol-2-yl)-2, 5-diphenyl-2H-tetrazolium bromide] assay was used to measure the proliferation of OS-732. 2 days after transfection, the incubated medium was replaced with new one and the cells was incubated for another 4 hours. And then added $50 \mu \mathrm{L}$ of DMSO into each well. A plate reader was used to measure the $\mathrm{A} 540$ of each sample after incubating for $10 \mathrm{~min}$ at $37^{\circ} \mathrm{C}$. Each test was repeated three times.

\section{Luciferase assay}

The 3'-UTR of BMP6 was amplified through PCR with carefully designed primers, then the PCR products were inserted into the pGL3 (Promega, Madison, WI, USA), and the 3'-UTR of BMP6 mutagenesis was inserted using site directed mutagenesis (Stratagene). And QIAGEN plasmid purification kit (QIAGEN, Hilden, Germany) was used to prepare the plasmids for transfection according to the manufacturer's recommendation. Lipofectamine 2000 (Invitrogen, Carlsbad, CA, USA) was used to transiently transfect the OS-732 cells with the recombination plasmids in accordance with the manufacturer's protocol. The DualLuciferase Reporter Assay System (Promega, Madison, WI, USA) was used to measure the activities of Firefly and Renilla luciferase 24 hours after transfection based on the standard protocol.

\section{Western blot analysis}

Pre-chilled PBS was used to wash the OS-732 cells, ice-cold lysis buffer (Biosharp, Hefei, China) including proteinase inhibitor (Roche, Indianapolis, IN, USA), $5 \mathrm{~g} / \mathrm{L}$ sodium deoxycholate, $0.2 \mathrm{~g} / \mathrm{L} \mathrm{NaN3}, 1 \%$ NP-40, $150 \mathrm{mmol} / \mathrm{L} \mathrm{NaCl}, 100 \mu \mathrm{g} / \mathrm{mL}, 0.1 \%$ SDS (sodium dodecyl sulfate), phenylmethylsulfonyl fluoride, 1 


\section{Cellular Physiology Cell Physiol Biochem 2017;41:2161-2170 \begin{tabular}{l|l|l}
\hline DOI: 10.1159/000475570 & $\begin{array}{l}\text { C } 2017 \text { The Author(s). Published by S. Karger AG, Basel } \\
\text { www.karger.com/cpb }\end{array}$ \\
\hline
\end{tabular}

$\mu \mathrm{g} / \mathrm{mL}$ aprotinin, and $50 \mathrm{mmol} / \mathrm{L}$ Tris- $\mathrm{HCl}(\mathrm{pH} 7.4)$ was used to lyse cells according to the manufacturer's introduction. The DC protein assay kit (Bio-Rad Laboratories, Hercules, CA, USA) was used to quantify the concentration of proteins, and hypotonic buffer (Invitrogen, California, USA) was used to dilute the proteins to an equal concentration. 4-20\% SDS-PAGE (sodium dodecyl sulfate polyacrylamide gel electrophoresis) was used to size-fractionat the protein, and then transferred to nitrocellulose membranes (Invitrogen, California, USA). TBST containing 5\% non-fat dry milk (Merck, Darmstadt, Germany) was used to block the membranes for 60 min. primary antibody against BMP6 (1:1,000; Santa Cruz Biotechnology, Beijing, China) was used to hybridize the membranes at $4^{\circ} \mathrm{C}$ for 12 hours. TBST was used to wash the membranes three times. HRP (horseradish peroxidase)-conjugated anti-mouse or rabbit secondary antibody (1:10000, Santa Cruz Biotechnology, Beijing, china) was used to hybridize the membranes for another 2 hours. TBST was used to wash the membranes three times. The enhanced chemiluminescence reagent (Thermo Fisher Scientific, Waltham, MA, USA) was used to detect the target protein by chemiluminescence, Quantity One software (Bio-Rad Laboratories) was used to quantify the optical density of the immunoblots based on the internal control ( $\beta$-actin). All experiments were carried out three times.

\section{Apoptosis analysis}

The flow cytometer (FACScalibure, Becton-Dickinson, France) was used to analyze the apoptosis of the OS-732 cells according to the manufacturer's protocol. Briefly, PI staining was performed on the fixed cells followed by flow cytometre using $488 \mathrm{~nm}$ to excite the cells and emission was observed at $585 \mathrm{~nm}$ to detect the extent of absorbance.

\section{Statistical analysis}

All data were shown as the mean $\pm \mathrm{SE}$ (standard error). One way analysis of variance (ANOVA) was used to estimate the comparisons among different groups, and then Dunnett's post hoc test was also used to estimate the comparisons among different groups. Two-way ANOVA was used to determine the statistical significance fordifferenttreatments, and then Bonferroni posthoctest was also used to determinethestatisticalsignificance for different treatments. Logistic regression analysis was used to determine the SNP of the genotyping.

\section{Results}

Demographic and clinicopathological characteristics of the patients enrolled in this study

We first collected 195 demographic and clinicopathological characteristics of patients enrolled in this study. We divided them into two groups by the genotype: $G G(n=124)$ and $\mathrm{GC}+\mathrm{CC}(\mathrm{n}=71)$. We collected information of age, gender, Total in-hospital time, smoking and alcohol consumption. Then we conducted $t$ test of age, gender and total in-hospital time and Chi-square test of smoking and alcohol consumption. Only total in-hospital time had significant difference between the two groups (approximately 2.5 times longer in GC/CC than GG), other variables are comparable. The details were shown in Table 1.

\section{MiR-124 was up-regulated in GG group}

We then collected 62 blood sample among these patients and extracted RNA from the blood sample. We then performed real time PCR, the results showed that the expression level of miR-124 was more than 2 times higher in GG group (Fig. 1) than GC and CC group.

BMP6 is a direct downstream target of $m i R-124-3 p$

Using publicly available databases, we searched candidate target genes

Table 1. Demographic and clinicopathological characteristics of the participants of this study

\begin{tabular}{|c|c|c|c|}
\hline Variables & $\begin{array}{c}\text { GG } \\
(n=124)\end{array}$ & $\begin{array}{l}\mathrm{GC}+\mathrm{CC} \\
(\mathrm{n}=71)\end{array}$ & $\mathrm{P}$ \\
\hline Age (years) & $35.3 \pm 2.3$ & $37.3 \pm 2.6$ & 0.08 \\
\hline Male gender $(\%)$ & 68.5 & 69.1 & 0.85 \\
\hline Total in-hospital time (days) & $18.6 \pm 3.2$ & $45.9 \pm 9.6$ & $<0.001$ \\
\hline \multicolumn{4}{|l|}{ Smoking } \\
\hline Non-smoking & $202 \quad(81.5)$ & $108 \quad(76.1)$ & 0.37 \\
\hline Smoking & $46 \quad(18.5)$ & $34 \quad(23.9)$ & \\
\hline \multicolumn{4}{|l|}{ Alcohol consumption } \\
\hline No & $136 \quad(54.8)$ & $72 \quad(50.7)$ & 0.58 \\
\hline Yes & $112 \quad(45.2)$ & $70 \quad(49.3)$ & \\
\hline
\end{tabular}




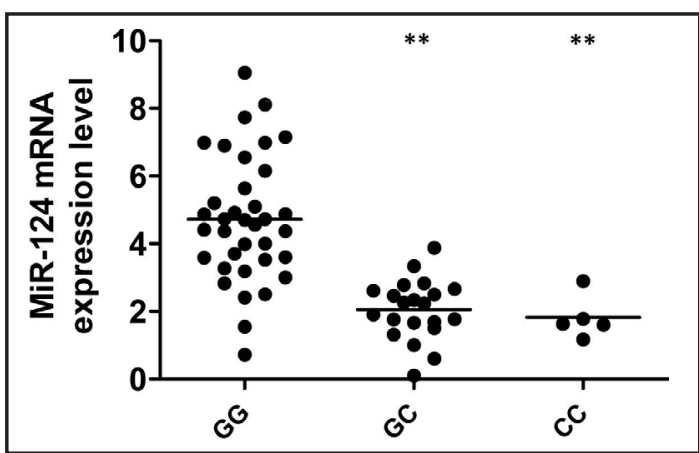

Fig. 1. The expression level of miR-124-3p was higher in GG group than GC and CC group ${ }^{* *} \mathrm{P}<0.01$ compared with the control).

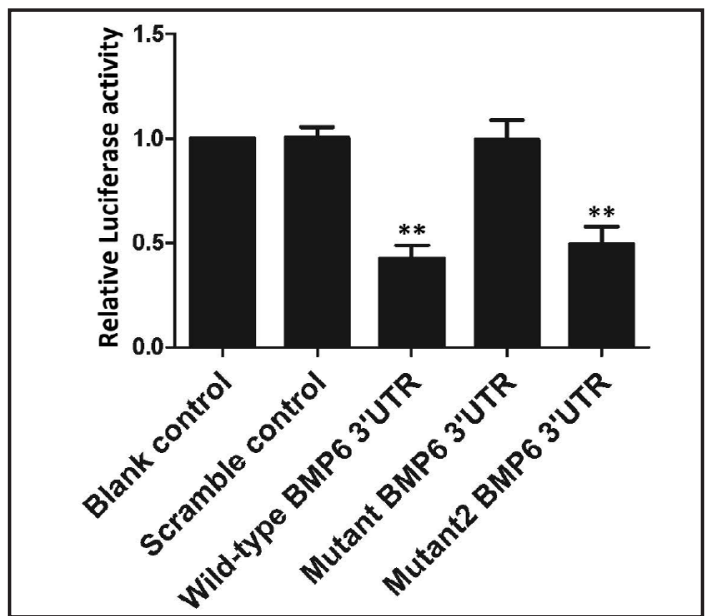

Fig. 3. Luciferase activity reporter assay was conducted to verify BMP6 as the direct target gene of miR-124-3p (** $\mathrm{P}<0.01$ compared with the control).

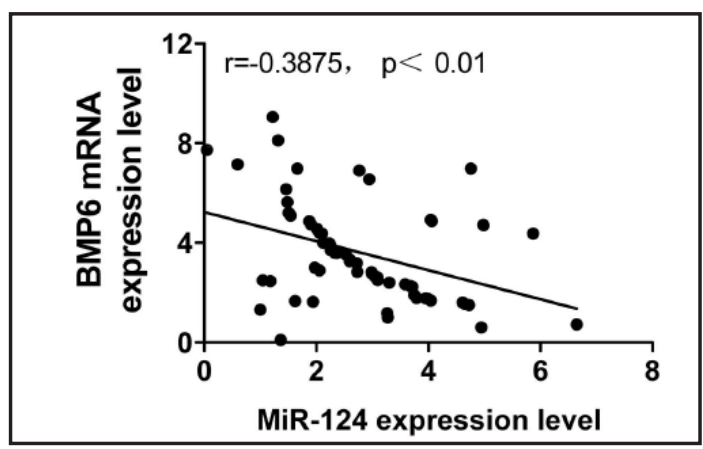

Fig. 4. The correlation between the expression level of miR-124-3p and BMP6 mRNA.

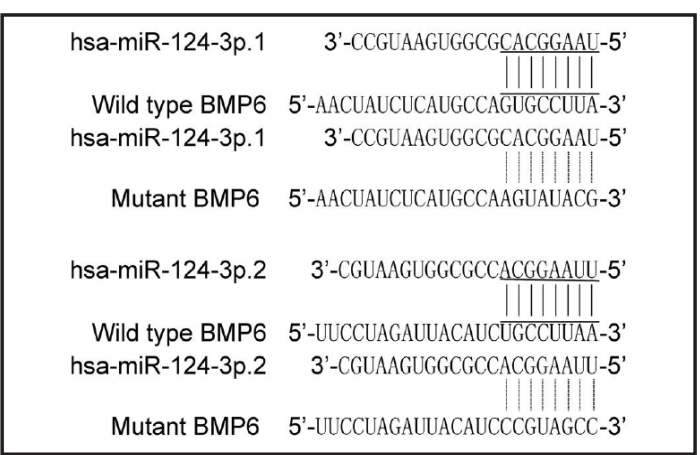

Fig. 2. BMP6 as the candidate target gene ofmiR124-3p in cells with the 'seed sequence' in the 3'UTR.

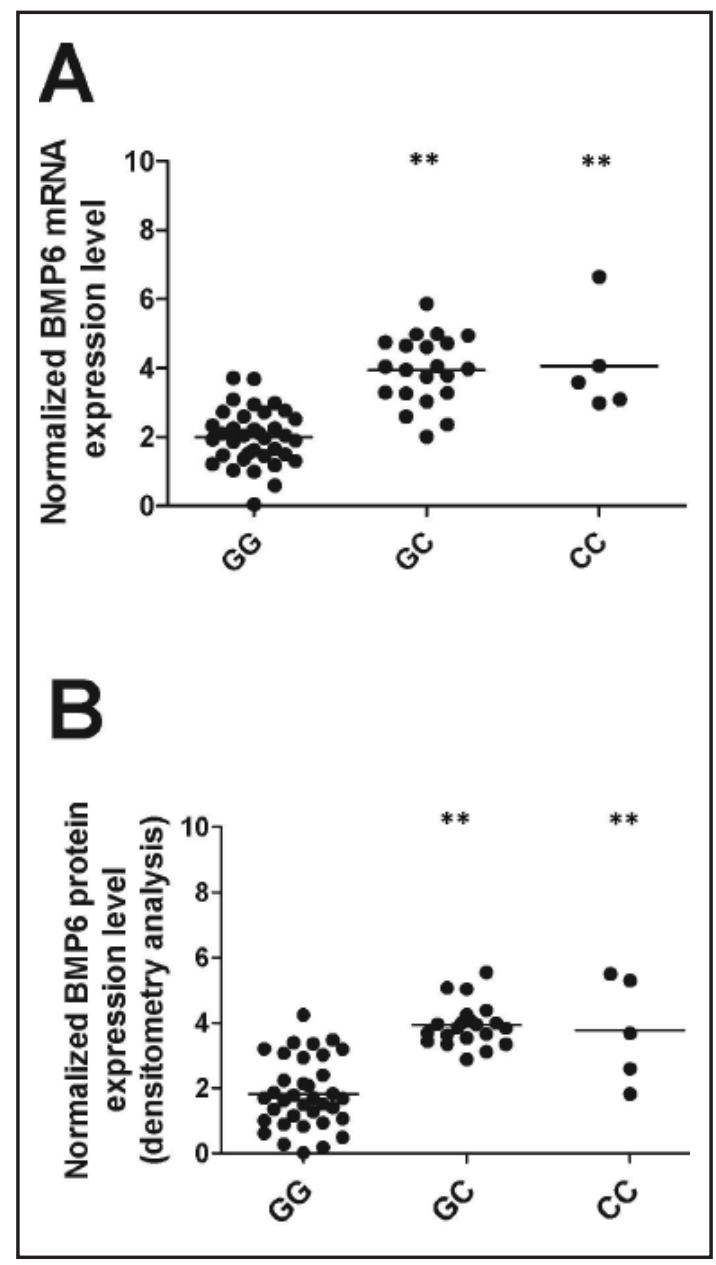

Fig. 5. The expression of BMP6 mRNA (Fig. 5A) and protein (Fig. 5B) decreased in GG group compared with GC and CC group ( ${ }^{* *} \mathrm{P}<0.01$ compared with the control).

of miR-124-3p, of all the candidates, BMP6 exhibited the most complementary structure with miR-124-3p (Fig. 2). We thus performed luciferase activity reporter assay in the cells to furthermore understand the regulatory relationship between miR-124-3p and BMP6.As shown in Fig. 3, the intensity of fluorescence from the cells co-transfected with miR-124-3p 
A
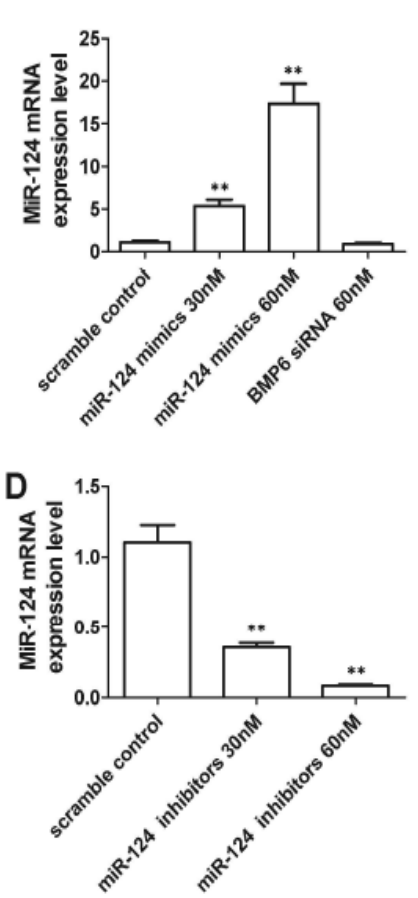

B

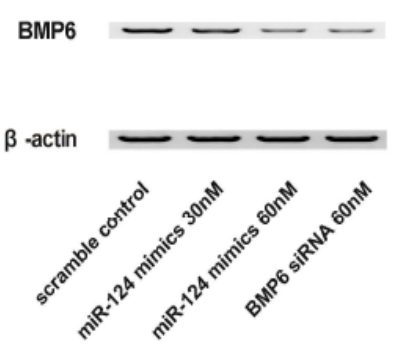

$\mathbf{E}$

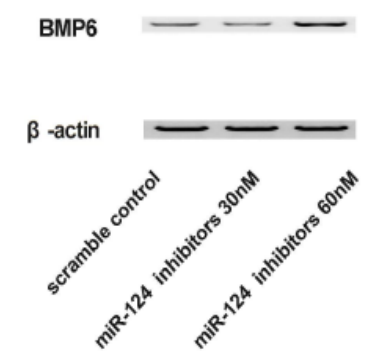

C

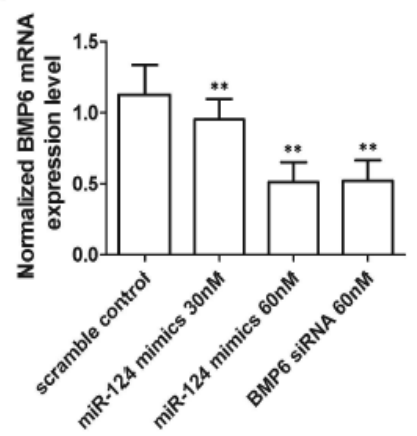

$\mathbf{F}$

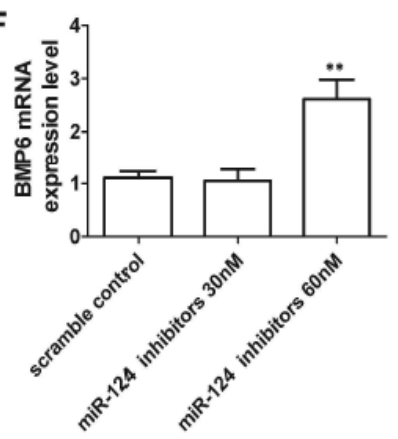

Fig. 6. When transfected with the cells with scramble control,miR-132 mimics(30nM and 60nM) ,BMP6 siRNA(60nM) and miR-124-3p inhibitors(30nM and 60nM), the expression level of miR-124-3p treated with miR-124-3p mimics(30nM and 60nM) and BMP6 siRNA increased(Fig. 6A), while cells treated with miR-124-3p inhibitors(30nM and 60nM) decreased(Fig. 6D); the expression level of BMP6 protein (Fig. 6B and E) and mRNA (Fig. 6C and F) treated with miR-124-3p mimics(30nM and 60nM) and BMP6 siRNA decreased, while cells treatedmiR-124-3p inhibitors(30nM and 60nM) increased ( ${ }^{* *} \mathrm{P}<0.01$ compared with the control).

and wild-type BMP6 or mutant2 BMP6 3'UTR decreased significantly (about 50\% lower, Fig. 3 ), while cells co-transfected with miR-124-3p and mutant BMP6 3'UTR were comparable compared with scramble control (Fig. 3). The results confirmed that BMP6 could bind to miR-124-3p, causing a significant decrease in luciferase activity in cells.

\section{Negative regulatory relationship between miR-124-3p and BMP6}

The blood sample of three different groups (GG, n=36, GC, $n=21, C C, n=5$ ) were used to further explore the impacts on the interaction between miR-124-3p and BMP6 3'UTR. We first analyzed the correlation between the expression level of miR-124-3p and BMP6 mRNA among the blood samples $(n=62)$, they showed negative regulatory relationship (Fig. $4, \mathrm{r}=-0.3875, \mathrm{p}<0.01$ ). We then performed real time PCR and densitometry analysis to detect the expression level of BMP6 mRNA and protein, we found them 2 times decreased in GG compared with GC and CC group (Fig. 5), confirming the negative regulatory relationship between miR-124-3p and BMP6. We further transfected with the cells with scramble control, miR-124-3p mimics (30nM and 60nM), BMP6 siRNA (60nM) and miR-124-3p inhibitors (30nM and 60nM). 48 hours after transfection, as shown in Fig. 6, compared with scramble control, up-regulation of miR-214-3p significantly decreased the expression of BMP6 mRNA and protein in a stepwise manner (Fig. 6B and C). We also found reduced the expression of BMP6 in the cells in a dose dependent manner; While down-regulation of miR-214-3p significantly increased the expression of BPM6 mRNA and protein level in the cells transfected with $60 \mathrm{nM}$ miR-124-3p inhibitors but not in $30 \mathrm{nM}$ (Fig. 6E and F). The observations indicated that miR-124-3p negatively regulated the expression of BMP6. 


\section{Cellular Physiology Cell Physiol Biochem 2017;41:2161-2170 \begin{tabular}{ll|l} 
DOI: 10.1159/000475570 & a 2017 The Author(s). Published by S. Karger AG, Basel \\
www.karger.com/cpb
\end{tabular}

Fig. 7. Cells transfected with miR124-3p inhibitors showed evidently reduced viability (Fig. 7A), while cells transfected withmiR-124-3p mimics and BMP6 siRNA showed higher viability; cells transfected with miR-124-3p mimics and BMP6 siRNA, reduced apoptosis while cells transfected with miR-124-3p inhibitors promoted apoptosis (Fig. 7B) (** $\mathrm{P}<0.01$ compared with the control).



MiR-124 and BMP6 interfered with the viability in cells

To further validate whether BMP6 is a functional target of miR-124-3p, we transfected with cells with scramble control, 30 and $60 \mathrm{nM}$ miR-124-3p mimics, 60nM BMP6 siRNA and 30 and $60 \mathrm{nM}$ miR-124-3p inhibitors. The results showed that cells treated with 60 nM miR-124-3p mimics and 60 nM BMP6 siRNA displayed evidently up-regulated viability (approximately 2.5 times elevation, Fig. 7A), and transfection of $30 \mathrm{nM}$ miR-124-3p mimics or inhibitors had minimal effect on viability. Meanwhile, the cells transfected with $60 \mathrm{nM}$ miR-124-3p inhibitors reduced viability to about 30\% of the control (Fig. 7A), suggesting miR-124-3p positively interfered with the viability of cells and BMP6 negatively interfered with the viability of cells.

MiR-124 and BMP6 interfered with the apoptosis in cells

We then investigated the relative apoptosis of cells when transfected with scramble control, 30/60nM miR-124-3p mimics, 60nM BMP6 siRNA and 30/60nM miR-124-3p inhibitors. When transfected with miR-124-3p mimics and BMP6 siRNA, the number of live cells were less and the number of apoptotic cells were 2 times more than the scramble controls (Fig. 7B), and $30 \mathrm{nM}$ miR-124-3p mimics or inhibitors had minimal effect on apoptosis. Meanwhile, the cells transfected with $60 \mathrm{nM}$ miR-124-3p inhibitors showed about $50 \%$ less live cells and more apoptotic cells. The results indicated miR-124-3p suppressed apoptosis and BMP6 promoted apoptosis.

\section{Discussion}

Fracture healing is a complex biological process involving four stages: inflammatory response, soft callus formation, hard callus formation, and bone remodeling [15]. The regeneration process of fracture healing is precisely organized by hormones, cytokines, chemokines, growth factors, and other regulators in each stage [16]. MiR-124-3p is commonly down-regulated in several types of cancer. Aberrant expression of miR-124-3p is related to methylation status of its locus [17]. In hepatocellular carcinoma, miR-124-3p is involved in an inflammatory feedback loop where it suppresses the expression of IL-6R and inhibits STAT3 activation. Systemic administration of miR-124-3p prevents hepatocellular carcinogenesis by inducing tumor-specific apoptosis [18]. In glioblastoma, miR-124-3p is implicated in T-cell-mediated antitumor immune response [19]. It has also been shown that miR-124-3p is significantly down-regulated in colon cancer patients with $\geq 5$-year survival compared to patients with $<5$ year survival. The level of miR-124-3p is associated with poor prognosis of CRC [20]. Zhang et al. also reported that miR-124-3p is significantly down-regulated in colon cancer tissue as compared with that of adjacent non-tumor colorectal tissue [21]. miR-124-3p has also been shown to directly suppress iASPP (inhibitor of apoptosis stimulating protein of p53) protein expression and up-regulate NF- $\kappa B$ level, which suppresses cell proliferation of colon cancer cells [22]. Over-expression of miR-124-3p leads to increased apoptosis of colon 


\section{Cellular Physiology Cell Physiol Biochem 2017;41:2161-2170 \begin{tabular}{l|l} 
DOI: 10.1159/000475570 & O 2017 The Author(s). Published by S. Karger AG, Basel \\
www.karger.com/cpb
\end{tabular} \\ Zou et al.: Rs531564 Polymorphism is Associated with Fracture Healing}

cancer cells and reduces tumor growth in vitro and in vivo [21]. In this study, we searched the publicly available databases, such as bioinformatics algorithms TargetScan (http:// www.targetscan.org/) and miRanda (http://www.microrna.org/microrna/home.do) to study candidate target genes of miR-124-3p-3p, of all the candidates, BMP 6 exhibited the most complementary structure with miR-124-3p-3p, and further confirmed using luciferase assay, we conducted the luciferase report with wild-type BMP6 3'UTR, mutant1 BMP6 3'UTR and mutant2 BMP6 3'UTR, we found that luciferase activity of the cells co-transfected with wild type BMP6 3'UTR was lower than those transfected with the scramble controls, but comparable with the cell transfected with mutant2 BMP6 3'UTR, while the introduction of mutant1 BMP6 with the potential "seed sequence" in the 3'TUR of BMP6 comparable with the scramble control and blank control.

Bone morphogenetic proteins (BMPs) is composed of a series of proteins that are members of the TGF-b superfamily. Several members of the family are key molecules in events such as odontogenesis and limb bud development [23-25]. BMPs in growth plate are also believed to promote maturation, as well as programmed cell death, or apoptosis [23, 26-28]. Recent research reports have focused on the distribution and role of BMPs in normal growth plate cell dynamics, which is the prototypical model of endochondral ossification [29]. BMP6-expression is significantly lower in proliferating non-malignant (PPCs) or malignant (HMCLs) cells and correlates inversely with the staging of the disease. In vitro, BMP6 significantly inhibits proliferation of HMCLs as well as primary MMCs and induces apoptosis in a time-dependent manner [30]. In this study, we investigated the different expression level of BMP6 mRNA and protein in different genotyping groups, and found the BMP6 mRNA and protein levels decreased in GG compared with GC and CC group. We further transfected with the cells with scramble control, miR-124-3p mimics (30nM and 60nM), BMP6 siRNA (60nM) and miR-124-3p inhibitors (30nM and 60nM), and found that the BMP6 mRNA and protein of cells were down-regulated following transfection with 30nM miR-124-3p mimics and, and ever lower when transfection with 60nM miR-124-3p mimics and 60nM siRNA BMP6, on the contrary, the BMP6 mRNA and protein of cells were remarkably up-regulated following transfection with 30nM miR-124-3p inhibitors and, and ever higher when transfection with $60 \mathrm{nM}$ miR-124-3p inhibitors. Moreover, we investigated the effect of miR-124-3p on cell viability and apoptosis, and we found that apoptosis of the cells transfected with miR-124$3 p$ mimics, BMP6 siRNA were reduced, while promoted subsequent to transfected with and miR-124-3p inhibitors compared with scramble controls.

MiRNA polymorphisms have been shown to influence the processing and expression of miRNAs, thereby altering target gene expression and influencing the development of diseases [31, 32]. One such polymorphism, miR-124-3p rs531564 (C > G), has been shown to effect the expression of mature miR-124-3p [33]. In this study, we performed westernblot and real-time PCR and revealed the expression of level of miR-124-3p was evidently up-regulated in GG group (Fig. 1) than GC and CC group. Furthermore, we enrolled a total of 195 participants for our research and the genotype of each participant was determined: GG $(n=124)$ and $G C+C C(n=71)$, and found that total in-hospital time was significantly longer in $\mathrm{GC}$ or CC group compared with GG group.

\section{Conclusion}

These data demonstrated that the expression of miR-124-3p was associated with the healing of metaphyseal fracture of distal tibia, and the presence of rs531564 polymorphism downregulated the expression of miR-124-3p increased the in-hospital stay time of the patients of metaphyseal fracture of distal tibia. Rs531564 polymorphism might be able to function as a biomarker of fracture healing of metaphyseal fracture of distal tibia. 


\section{Cellular Physiology Cell Physiol Biochem 2017;41:2161-2170 \begin{tabular}{ll|l} 
and Biochemistry & $\begin{array}{l}\text { DOI: 10.1159/000475570 } \\
\text { Published online: April 21, } 2017\end{array}$ & $\begin{array}{l}\text { (c) } 2017 \text { The Author(s). Published by S. Karger AG, Basel } \\
\text { www.karger.com/cpb }\end{array}$
\end{tabular}

\section{Disclosure Statement}

No conflict of interest declared.

\section{References}

Bechtle J: Osteoporosis risk reduction and bone health. Nurs Womens Health 2013;17:245-251.

Phillips AM: Overview of the fracture healing cascade. Injury 2005;36 Suppl 3:S5-7.

Gaston MS, Simpson AH: Inhibition of fracture healing. J Bone Joint Surg Br 2007;89:1553-1560.

Moriishi T, Fukuyama R, Miyazaki T, Furuichi T, Ito M, Komori T: Overexpression of BCLXL in Osteoblasts Inhibits Osteoblast Apoptosis and Increases Bone Volume and Strength. J Bone Miner Res 2016;31:13661380.

-5 Hu L, Han J, Yang X, Wang Y, Pan H, Xu L: Apoptosis repressor with caspase recruitment domain enhances survival and promotes osteogenic differentiation of human osteoblast cells under Zoledronate treatment. Mol Med Rep 2016;14:3535-3542.

6 Guo C, Yang XG, Wang F, Ma XY: IL-1alpha induces apoptosis and inhibits the osteoblast differentiation of MC3T3-E1 cells through the JNK and p38 MAPK pathways. Int J Mol Med 2016;38:319-327.

7 Gibson G: Active role of chondrocyte apoptosis in endochondral ossification. Microsc Res Tech 1998;43:191-204.

8 Gibson GJ, Kohler WJ, Schaffler MB: Chondrocyte apoptosis in endochondral ossification of chick sterna. Dev Dyn 1995;203:468-476.

-9 Yang XC, Deng ZH, Wen T, Luo W, Xiao WF, Zhao RB, Li YS: Network Meta-Analysis of Pharmacological Agents for Osteoporosis Treatment and Fracture Prevention. Cell Physiol Biochem 2016;40:781-795.

10 Lee RC, Feinbaum RL, Ambros V: The C. elegans heterochronic gene lin-4 encodes small RNAs with antisense complementarity to lin-14. Cell 1993;75:843-854.

11 He L, Hannon GJ: MicroRNAs: small RNAs with a big role in gene regulation. Nat Rev Genet 2004;5:522-531.

12 Reinhart BJ, Weinstein EG, Rhoades MW, Bartel B, Bartel DP: MicroRNAs in plants. Genes Dev 2002;16:1616-1626.

13 Bartel DP: MicroRNAs: target recognition and regulatory functions. Cell 2009;136:215-233.

14 Huang J, Peng J, Cao G, Lu S, Liu L, Li Z, Zhou M, Feng M, Shen H: Hypoxia-Induced MicroRNA-429 Promotes Differentiation of MC3T3-E1 Osteoblastic Cells by Mediating ZFPM2 Expression. Cell Physiol Biochem 2016;39:1177-1186.

15 Marsell R, Einhorn TA: The biology of fracture healing. Injury 2011;42:551-555.

16 You L, Pan L, Chen L, Gu W, Chen J: MiR-27a is Essential for the Shift from Osteogenic Differentiation to Adipogenic Differentiation of Mesenchymal Stem Cells in Postmenopausal Osteoporosis. Cell Physiol Biochem 2016;39:253-265.

17 Wilting SM, van Boerdonk RA, Henken FE, Meijer CJ, Diosdado B, Meijer GA, le Sage C, Agami R, Snijders PJ, Steenbergen RD: Methylation-mediated silencing and tumour suppressive function of hsa-miR-124 in cervical cancer. Mol Cancer 2010;9:167.

-18 Hatziapostolou M, Polytarchou C, Aggelidou E, Drakaki A, Poultsides GA, Jaeger SA, Ogata H, Karin M, Struhl K, Hadzopoulou-Cladaras M, Iliopoulos D: An HNF4alpha-miRNA inflammatory feedback circuit regulates hepatocellular oncogenesis. Cell 2011;147:1233-1247.

19 Wei J, Wang F, Kong LY, Xu S, Doucette T, Ferguson SD, Yang Y, McEnery K, Jethwa K, Gjyshi O, Qiao W, Levine NB, Lang FF, Rao G, Fuller GN, Calin GA, Heimberger AB: miR-124 inhibits STAT3 signaling to enhance T cell-mediated immune clearance of glioma. Cancer Res 2013;73:3913-3926.

-20 Sun Y, Zhao X, Zhou Y, Hu Y: miR-124, miR-137 and miR-340 regulate colorectal cancer growth via inhibition of the Warburg effect. Oncol Rep 2012;28:1346-1352.

21 Zhang J, Lu Y, Yue X, Li H, Luo X, Wang Y, Wang K, Wan J: MiR-124 suppresses growth of human colorectal cancer by inhibiting STAT3. PLoS One 2013;8:e70300.

22 Liu K, Zhao H, Yao H, Lei S, Lei Z, Li T, Qi H: MicroRNA-124 regulates the proliferation of colorectal cancer cells by targeting iASPP. Biomed Res Int 2013;2013:867537.

23 Edson MA, Nagaraja AK, Matzuk MM: The mammalian ovary from genesis to revelation. Endocr Rev 2009;30:624-712. 


\section{Cellular Physiology Cell Physiol Biochem 2017;41:2161-2170 and Biochemistry Published 1159/000475570 $2017 \quad \begin{aligned} & \text { O 2017 The Author(s). Published by S. Karger AG, Basel } \\ & \text { www.karger.com/cpb }\end{aligned}$ Zou et al.: Rs531564 Polymorphism is Associated with Fracture Healing}

24 Yi SE, LaPolt PS, Yoon BS, Chen JY, Lu JK, Lyons KM: The type I BMP receptor BmprIB is essential for female reproductive function. Proc Natl Acad Sci U S A 2001;98:7994-7999.

-25 Edson MA, Nalam RL, Clementi C, Franco HL, Demayo FJ, Lyons KM, Pangas SA, Matzuk MM: Granulosa cell-expressed BMPR1A and BMPR1B have unique functions in regulating fertility but act redundantly to suppress ovarian tumor development. Mol Endocrinol 2010;24:1251-1266.

26 Middlebrook BS, Eldin K, Li X, Shivasankaran S, Pangas SA: Smad1-Smad5 ovarian conditional knockout mice develop a disease profile similar to the juvenile form of human granulosa cell tumors. Endocrinology 2009;150:5208-5217.

-27 Shimasaki S, Moore RK, Otsuka F, Erickson GF: The bone morphogenetic protein system in mammalian reproduction. Endocr Rev 2004;25:72-101.

28 Otsuka F: Multiple endocrine regulation by bone morphogenetic protein system. Endocr J 2010;57:3-14.

29 Knight PG, Glister C: TGF-beta superfamily members and ovarian follicle development. Reproduction 2006;132:191-206.

-30 Ro TB, Holt RU, Brenne AT, Hjorth-Hansen H, Waage A, Hjertner O, Sundan A, Borset M: Bone morphogenetic protein-5, -6 and -7 inhibit growth and induce apoptosis in human myeloma cells. Oncogene 2004;23:3024-3032.

- 31 Srivastava K, Srivastava A: Comprehensive review of genetic association studies and meta-analyses on miRNA polymorphisms and cancer risk. PLoS One 2012; 7:e50966.

-32 Wang X, Tang S, Le SY, Lu R, Rader JS, Meyers C, Zheng ZM: Aberrant expression of oncogenic and tumorsuppressive microRNAs in cervical cancer is required for cancer cell growth. PLoS One 2008;3:e2557.

33 Qi L, Hu Y, Zhan Y, Wang J, Wang BB, Xia HF, Ma X: A SNP site in pri-miR-124 changes mature miR124 expression but no contribution to Alzheimer's disease in a Mongolian population. Neurosci Lett 2012;515:1-6. 